\title{
Observations of Binary Stars with the Differential Speckle Survey Instrument. VII. Measures from 2010 September to 2012 February at the WIYN Telescope
}

\author{
Elliott P. Horch ${ }^{1,9,12}$, Dana I. Casetti-Dinescu ${ }^{1}$, Matthew A. Camarata ${ }^{1,10,12}$, Akbar Bidarian ${ }^{1,12}$, \\ William F. van Altena ${ }^{2,12}$, William H. Sherry ${ }^{3,11}$, Mark E. Everett ${ }^{3}$, Steve B. Howell ${ }^{4,12}$, \\ David R. Ciardi ${ }^{5}$, Todd J. Henry ${ }^{6}$, Daniel A. Nusdeo ${ }^{7}$, and Jennifer G. Winters ${ }^{8}$ \\ ${ }^{1}$ Department of Physics, Southern Connecticut State University, 501 Crescent Street, New Haven, CT 06515, USA \\ horche2@southernct.edu, danacasetti@gmail.com, camarata@hhne.org, bidariana1@ southernct.edu \\ ${ }^{2}$ Department of Astronomy, Yale University, P.O. Box 208101, New Haven, CT 06520-8101, USA; william.vanaltena@yale.edu \\ ${ }^{3}$ National Optical Astronomy Observatory, 950 North Cherry Avenue, Tucson, AZ 85719, USA \\ william.sherry@paradisevalley.edu, everett@noao.edu \\ ${ }^{4}$ NASA Ames Research Center, Moffett Field, CA 94035, USA; steve.b.howell@nasa.gov \\ ${ }^{5}$ NASA Exoplanet Science Institute, California Institute of Technology, 770 South Wilson Avenue, \\ Mail Code 100-22, Pasadena, CA 91125, USA; ciardi@ipac.caltech.edu \\ ${ }^{6}$ RECONS Institute, Chambersburg, PA 17201, USA; thenry@astro.gsu.edu \\ ${ }^{7}$ Department of Physics and Astronomy, Georgia State University, 25 Park Place, Atlanta, GA 30302, USA; nusdeo@astro.gsu.edu \\ ${ }^{8}$ Harvard-Smithsonian Center for Astrophysics, 60 Garden Street, Cambridge, MA 02138, USA; jennifer.winters@cfa.harvard.edu \\ Received 2017 February 17; revised 2017 March 10; accepted 2017 March 10; published 2017 April 14
}

\begin{abstract}
We report on speckle observations of binary stars carried out at the WIYN Telescope over the period from 2010 September through 2012 February, providing relative astrometry for 2521 observations of 883 objects, 856 of which are double stars and 27 of which are triples. The separations measured span a range of $0.01-1.75$ arcsec. Wavelengths of 562, 692, and $880 \mathrm{~nm}$ were used, and differential photometry at one or more of these wavelengths is presented in most cases. 66 components were resolved for the first time. We also estimate detection limits at 0.2 and 1.0 arcsec for high-quality observations in cases where no companion was seen, a total of 176 additional objects. Detection limits vary based on observing conditions and signal-to-noise ratio, but are approximately 4 mag at 0.2 arcsec and $6 \mathrm{mag}$ at 1.0 arcsec on average. Analyzing the measurement precision of the data set, we find that the individual separations obtained have linear measurement uncertainties of approximately 2 mas, and photometry is uncertain to approximately $0.1 \mathrm{mag}$ in general. This work provides fundamental, well-calibrated data for future orbit and mass determinations, and we present three first orbits and total mass estimates of nearby K-dwarf systems as examples of this potential.
\end{abstract}

Key words: binaries: visual - techniques: high angular resolution - techniques: interferometric techniques: photometric

Supporting material: machine-readable tables

\section{Introduction}

Binary stars remain an important tool in furthering two fundamental areas of astrophysics: (1) they contribute to an understanding of stellar structure and evolution by providing empirically determined masses of stars, and (2) when used statistically, observationally determined separations, magnitude differences, and orbital parameters can yield information important for understanding the relative importance of star (and by extension, exoplanet) formation mechanisms. Speckle imaging has contributed in both areas over its long history, and indeed the technique has seen a resurgence in recent years due to the more widespread use of electron-multiplying CCDs

\footnotetext{
9 Adjunct Astronomer, Lowell Observatory, 1600 West Mars Hill Road, Flagstaff, AZ 86001, USA.

${ }^{10}$ Current Address: Hebrew High School of New England, 30 Bloomfield Avenue, West Hartford, CT 06117, USA.

${ }^{11}$ Current Addresses: Physical Sciences Department, Glendale Community College, 6000 West Olive Avenue, Glendale, AZ 85302, USA and Eureka Scientific, Inc., 2452 Delmer Street, Suite 100, Oakland, CA 946023017, USA.

12 Visiting Astronomer, Kitt Peak National Observatory, National Optical Astronomy Observatory, which is operated by the Association of Universities for Research in Astronomy, Inc. (AURA) under cooperative agreement with the National Science Foundation.
}

(EMCCDs) in speckle work (Tokovinin \& Cantarutti 2008; Docobo et al. 2010; Horch et al. 2011; Balega et al. 2013). In addition to opening new parameter space for the measurement of binary systems due to their sensitivity and readout speed, these devices have enabled the use of speckle imaging for faint (stellar) companion detection for stars thought to host exoplanets (Howell et al. 2011, 2016; Furlan et al. 2017; Hirsch et al. 2017). Speckle imaging has been used together with adaptive optics to determine if a stellar companion is present, and if so, what its brightness relative to the primary star is (e.g., Crossfield et al. 2016), which is an important consideration in deriving the correct radius of the exoplanet (Ciardi et al. 2015). Adaptive optics and speckle imaging are essentially complementary in this regard because the bulk of adaptive optics observations have relatively shallow detection limits within 0.2 arcsec, due to the fact that the wavelengths of observation are usually in the infrared; in contrast, speckle imaging provides diffraction-limited imaging over a very small field of view, but in visible wavelengths. Therefore, the detection limits close to the target star are often deeper for a comparable telescope aperture, something that enables astrophysics with close binary stars as well as exoplanet host star vetting. 
Our past work at the WIYN ${ }^{13}$ telescope has involved both surveys of binary stars and exoplanet host stars. In particular, earlier papers in this series have detailed our complete survey of Hipparcos double stars (HDSs) and suspected doubles, which began in the late 1990s (Horch et al. 1999), was active over the next 15 years (e.g., Horch et al. 2012, 2015b, and references therein), and has continued through to the present work. This program has contributed a large amount of relative astrometry and photometry data as well as about two dozen orbits and total mass estimates. Some of the smallest separation systems that we have successfully observed at WIYN have had separations below the diffraction limit of $\sim 50$ mas at $692 \mathrm{~nm}$ (Horch et al. 2011b), which has led to follow-up observations at larger telescopes and the determination of a handful of short-period orbits, with periods of a few months to a few years (Horch et al. 2015a). In the area of exoplanet host star observations, we have developed a methodology for companion detection from reconstructed images obtained from speckle data (Horch et al. 2011; Howell et al. 2011), and used this to estimate the fraction of Kepler exoplanet hosts that also have a stellar companion (Horch et al. 2014). We have also set limits on stellar companions for exoplanets with eccentric orbits (Kane et al. 2014) and developed techniques for assessing whether a companion star is likely to be gravitationally bound to its primary (Everett et al. 2015; Teske et al. 2015).

The observations described hererepresent our seventh (and so far largest) installment of relative astrometry and photometry of binary stars. This brings the total number of measures contributed by the WIYN speckle program to over 8000 . We anticipate that after this there will be one more large group of measures to be published from data taken at WIYN on the general binary survey work that will cover the time frame of mid-2012 to the end of 2013. From that point forward, the observing lists changed significantly to accommodate new scientific goals including more exoplanet host-star observations, and less time was spent on binary-star surveys. Most HDSs and suspected doubles within $250 \mathrm{pc}$ of the solar system and observable from WIYN had been measured by our program by the end of 2013, and many of the binaries amenable to providing astrophysical information within the next decade had been identified. Since that point, the focus has been on understanding multiplicity as a function of spectral type and metallicity, and in following up on promising systems for mass estimates. This paper, while mainly contributing to the earlier survey work, also moves the direction to these new projects involving star formation and multiplicity statistics by providing first orbits for three K-dwarf systems.

\section{Observations}

The observations were carried out over six runs at the WIYN telescope, specifically, 2010 September 17-21, 2010 October 23-26, 2011 June 11-16, 2011 September 7-11, 2011 December 10-11, and 2012 February 4-8. In each case, an observing list was constructed primarily from HDSs and Hipparcos suspected doubles (ESA 1997), double-lined spectroscopic binary stars identified in the Geneva-Copenhagen spectroscopic survey (Nordström et al. 2004), and stars we have previously found to be double in our own program and reported in earlier papers in this series.

\footnotetext{
13 At the time of these observations, the WIYN Observatory was a joint facility of the University of Wisconsin-Madison, Indiana University, Yale University, and the National Optical Astronomy Observatories.
}

For all observations here, the Differential Speckle Survey Instrument (DSSI) was used (Horch et al. 2009). The instrument can mount to either of the Nasmyth ports of the WIYN telescope and takes speckle observations in two filters simultaneously. The DSSI observing program at WIYN began in 2008, and the instrument was upgraded to use two electron-multiplying CCD cameras in 2010 January. More recently, DSSI also been used at Lowell Observatory's Discovery Channel Telescope (DCT), and at both the Gemini north and Gemini south telescopes.

The typical observing sequence was to record $100040 \mathrm{~ms}$ frames of a target star in two filters simultaneously. Each frame consists of a $128 \times 128$ pixel subarray on the chip, which represents a field of view of approximately $2.8 \times 2.8 \mathrm{arcsec}$. This was followed by a similar sequence of a bright (5th magnitude) unresolved star that is within a few degrees of the science target. The latter serves as an estimate of the speckle transfer function, which is then used in the data analysis. For fainter targets, we sometimes took more than 1000 frames; for example, for a 12th or 13th magnitude star, we would typically take $3000-5000$ frames, depending on seeing conditions. To make observations more efficient, we would observe up to several science targets that were close together on the sky in a sequence of increasing R.A., working in a single bright unresolved calibrator at the appropriate moment based on its R.A. This star was then used as the calibrator for all science targets in the group. The resulting data files were stored in FITS format, each object having two files, one for each wavelength used.

\section{Data Reduction and Analysis}

\subsection{Determination of the Pixel Scale and Orientation}

The pixel scale and orientation were determined in a similar fashion to our previous work at WIYN. A slit mask that mounts to the tertiary baffle support structure on the telescope is used. Once in place, this allows us to take speckle data of a very bright unresolved star (typically 2nd or 3rd magnitude) through the slits. The resulting images show a series of superimposed fringes of different spatial frequencies. If a speckle sequence is taken and the spatial frequency power spectrum of the ensemble of images calculated, these fringes map to sharp, linear features in the Fourier plane that can be fitted with high precision. Using a measure of the separation of the slits, the distance from the mask to the focal plane, the wavelength of the light used, and the focal ratio of the telescope, the spatial frequencies obtained from the fit in terms of cycles per pixel can be translated into cycles per arcsecond. Upon inverting, this then yields the scale in arcseconds per pixel.

Three of the four quantities needed for the calibration are straightforward to measure: observatory staff have measured the distance from the baffle support to the nominal focal plane to much better than $0.1 \%$. The slit spacing has been measured directly from the mask to a similar precision by one of us (E.H.). The focal ratio of the telescope is of course well known. The final quantity, the effective wavelength of the observation, is a combination of the filter and atmospheric transmission, quantum efficiency of the detector, and the spectrum of the star being observed. In order to get the best possible value of this quantity, we use the Pickles (1998) spectral library, interpolating to the correct spectral type when needed, and we combine this with vendor-provided curves for quantum efficiency and filter transmission. A Kitt Peak atmospheric transmission curve is available from the observatory website. By simulating the wavelength distribution that is in fact detected, we can then 


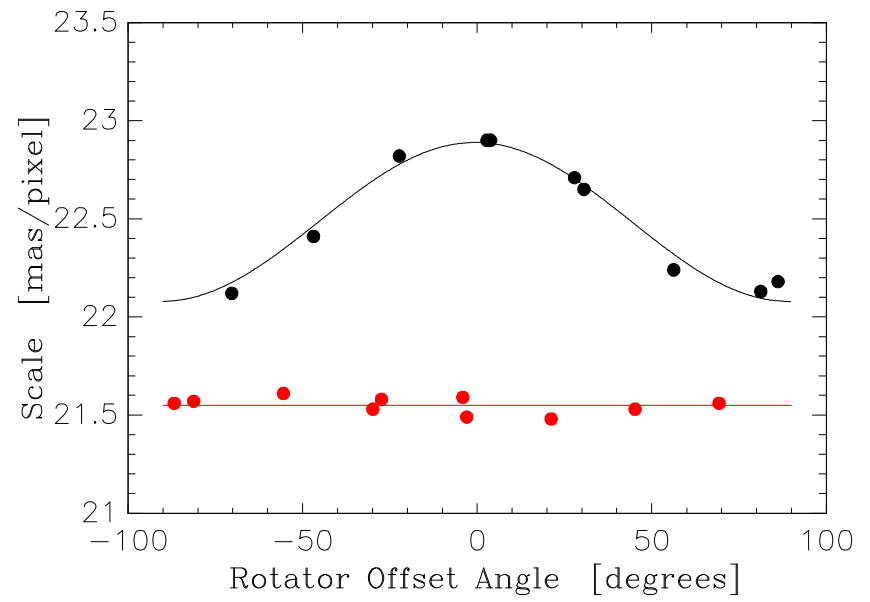

Figure 1. Scale determination as a function of instrument rotator angle. In this case, HR 5107 was observed through the slit mask in 2011 June. The red points are from Channel A (using the $692 \mathrm{~nm}$ filter), and the black points are from Channel B (using the $880 \mathrm{~nm}$ filter).

estimate the effective wavelength as the average value of the photon flux of this distribution.

As we have reported in earlier papers, the channel of DSSI where light reflects off of the dichroic element inside the camera (which we refer to as "Channel B") exhibits a slight variation in pixel scale as a function of position angle. This distortion is consistent with what would be expected if a reflective surface in the instrument, such as the dichroic, were not oriented at exactly at $45^{\circ}$ relative to the incoming optical axis. We have extensively mapped out the effect by taking slit mask data at different orientations of the mask relative to the detector axes. This is easily accomplished by rotating the instrument adapter subsystem on the Nasmyth port. Figure 1 shows an example of the results in scale obtained as a function of rotator offset angle. In the case of the Channel B data, the variation is about $\pm 2 \%$ from the mean, a typical result at WIYN. In contrast, the Channel A data show no clear evidence of such an effect, and so we simply average all of the values obtained for a given run to obtain the final value of the scale for that channel. The standard error of these values has an average of 0.026 mas/pixel; we view this as a lower limit to the uncertainty in the scale, as it is obtained from a single sequence of observations on only one star.

One possible source of systematic error in the scale determination is the effective wavelength of the slit mask observations, if the filter transmission curves or the stellar spectrum selected from the Pickles library are not good models of the observations. In order to investigate the effect of color variation on the scale determination, we observed two stars with the slit mask in a single run on three occasions. The stars were chosen to have very different spectral types, either A and $\mathrm{M}$ or $\mathrm{A}$ and $\mathrm{K}$. If we subtract the two scales obtained in a given channel, the difference should be zero if there is no systematic effect in color. The value obtained in these cases is $-0.036 \pm 0.023 \mathrm{mas} / \mathrm{pixel}$, showing that the systematic error due to the color calibration is at most very modest. A conservative total scale uncertainty can be obtained by adding this value in quadrature with the 0.026 mas/pixel figure from the internal repeatability mentioned above, resulting in $0.044 \mathrm{mas} / \mathrm{pixel}$, or about $0.2 \%$ of the nominal scale value.

The orientation angle between celestial coordinates and pixel axes is determined mainly by taking $1 \mathrm{~s}$ images of stars from The Bright Star Catalog (Hoffleit \& Jaschek 1982) and offsetting the telescope in different directions between exposures. By using the full area of the chip, we can obtain a sufficient baseline to measure the offset angle to $\sim 0.5$ in good conditions for a given run. Such a sequence of observations is usually taken nightly during the run, giving several measures per run, but if the sequence is judged to have been taken in poor seeing or under windy conditions, we sometimes removed a nightly sequence from further consideration. Using only runs where we have three or more sequences meeting the "high quality" criteria, we conclude that the standard error in offset angle has an average value of 0.38 per run when combining information in both channels.

Regardless of the offset angle between pixel axes and celestial coordinates, Channels A and B should be mirror images of one another about some symmetry axis that is nearly aligned with one of the pixel axes. To establish this small internal offset angle, we sought to combine both the A and B results to get a single value of this offset with the lowest random uncertainty. The mask files can be used in this regard to establish the axis of symmetry relative to the pixel axes to high precision by assuming that any offset angle in Channel B is the same relative to this axis as in Channel A, although opposite in direction. Thus, if the orientation angles obtained are merely averaged, then the result is an estimate of the angle made between the axis of symmetry and the pixel axes. For each run, we determined the mean value of this angle and then averaging these results over all runs; we find a value of $\theta_{0}=0.12 \pm 0.13$. This figure is used with all runs in our analysis here. Then, the offset angle between this symmetry axis and celestial coordinates in Channel $\mathrm{A}$ is given by $+\theta_{1}$, and $-\theta_{1}$ in Channel $\mathbf{B}$, where $\theta_{1}$ is the average of the absolute value of the offset obtained using the $1 \mathrm{~s}$ offset images. In this way, the angle between the symmetry axis and the pixel axes remains the same from run to run, as it is most likely internal to the DSSI instrument, but the size of the offset in both directions relative to that axis varies from run to run, as that is most likely related to the small variations in the zero point of the instrument rotator and the mounting of the instrument on the telescope, which may change from run to run. Given this, the total uncertainty for the angle is obtained by adding the uncertainties in the determination of the symmetry axis and that of the $1 \mathrm{~s}$ images; we find $\sqrt{0.38^{2}+0.13^{2}}=00^{\circ} 40$ as the final angle uncertainty from this calibration. This is assumed for all runs, as shown in Table 1.

\subsection{Speckle Data Reduction}

While our reduction method from raw speckle frames remains the same as in previous papers in this series, a brief summary is provided here for convenience. The frames are debiased and the autocorrelation of each frame in the observation is computed. These are then summed. The same computation is performed on the speckle frames of the calibration point source. Both autocorrelations are Fourier transformed, and then the result for the science target is divided by that of the point source to deconvolve the speckle transfer function from the observation. The square root of this function is then computed, which forms the diffraction-limited modulus estimate for the science target's Fourier transform.

Lohmann et al. (1983) first described how phase information of the object's Fourier transform is contained in the summed triple correlation function of speckle images, and its Fouriertransform pair, the image bispectrum. We compute near-axis sub-planes of the bispectrum for the science observation 
Table 1

Pixel Scale, Orientation, and Filters Used on Each Run

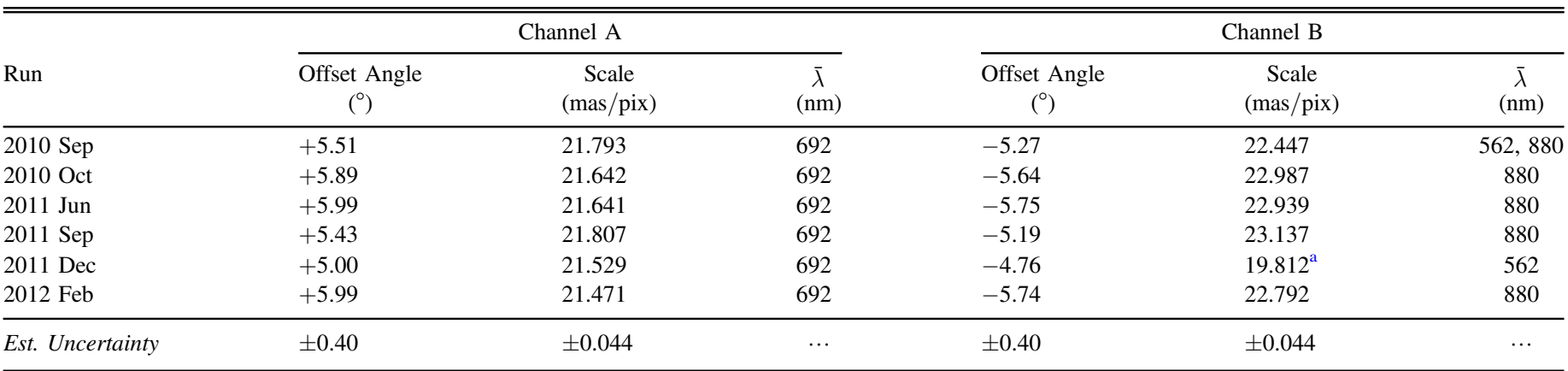

Note.

${ }^{a}$ A different optical configuration was used in this channel compared to the other runs.

Table 2

Double Star Speckle Measures

\begin{tabular}{|c|c|c|c|c|c|c|c|c|c|}
\hline $\begin{array}{l}\text { WDS } \\
(\alpha, \delta \mathbf{J} 2000.0)\end{array}$ & $\begin{array}{l}\mathrm{HR}, \mathrm{ADS} \\
\mathrm{HD}, \text { or DM }\end{array}$ & $\begin{array}{l}\text { Discoverer } \\
\text { Designation }\end{array}$ & HIP & $\begin{array}{c}\text { Date } \\
(2000+)\end{array}$ & $\begin{array}{l}\theta \\
\left(^{\circ}\right)\end{array}$ & $\begin{array}{c}\rho \\
\left({ }^{\prime \prime}\right)\end{array}$ & $\Delta m$ & $\begin{array}{c}\lambda \\
(\mathrm{nm})\end{array}$ & $\begin{array}{c}\Delta \lambda \\
(\mathrm{nm})\end{array}$ \\
\hline \multirow[t]{3}{*}{$00022-2052$} & HD 224961 & YSC 168 & 170 & 11.6837 & 39.8 & 0.1105 & 0.42 & 692 & 40 \\
\hline & & & & 11.6837 & 40.2 & 0.1156 & 0.88 & 880 & 50 \\
\hline & & & & 11.9430 & 37.0 & 0.1176 & 0.65 & 562 & 40 \\
\hline \multirow[t]{2}{*}{$00031+5228$} & HD 225064 & HDS 3 & 250 & 11.9431 & 333.5 & 0.3221 & 3.16 & 692 & 40 \\
\hline & & & & 11.9431 & 333.5 & 0.3184 & 2.89 & 562 & 40 \\
\hline \multirow[t]{2}{*}{$00067+0839$} & $\cdots$ & LSC 3 & 551 & 11.6864 & 15.6 & 0.5692 & 4.11 & 692 & 40 \\
\hline & & & & 11.6864 & 15.9 & 0.5676 & 3.08 & 880 & 50 \\
\hline \multirow[t]{4}{*}{$00071-1551$} & HD 233 & HDS 11 & 584 & 11.6837 & 253.1 & 0.4029 & 3.14 & 692 & 40 \\
\hline & & & & 11.6837 & 252.8 & 0.4054 & 2.71 & 880 & 50 \\
\hline & & & & 11.9430 & 253.3 & 0.4004 & 3.27 & 692 & 40 \\
\hline & & & & 11.9430 & 252.9 & 0.4049 & 3.54 & 562 & 40 \\
\hline \multirow[t]{2}{*}{$00085+3456$} & HD 375 & HDS 17 & 689 & 10.7143 & 81.0 & 0.1246 & 0.27 & 692 & 40 \\
\hline & & & & 10.7143 & 81.1 & 0.1238 & 0.32 & 562 & 40 \\
\hline
\end{tabular}

Notes.

${ }^{\mathrm{a}}$ Quadrant ambiguous.

${ }^{\mathrm{b}}$ Quadrant inconsistent with previous measures in the 4th Interferometric Catalog.

${ }^{c}$ These measures were previously published in Horch et al. (2011); this reanalysis should replace the previous values.

${ }^{\mathrm{d}}$ These measures were previously published in Appourchaux et al. (2015); this reanalysis should replace the previous values.

e This star is a member of the nearby K-dwarf sample discussed in the text.

(This table is available in its entirety in machine-readable form.)

following their methodology. The phase of the object's Fourier transform is then computed from these, using the relaxation algorithm of Meng et al. (1990). This is combined with the modulus estimate previously obtained to give the (complex) Fourier transform of the object. This is low-pass filtered with a two-dimensional Gaussian function, and inverse transformed. The result is a diffraction-limited reconstructed image of the object. This is used to identify secondary and tertiary components in the case of binaries and trinaries, and used in the case of non-detections to estimate the detection limit of the observation as described further in Section 3.4.

In the case of binary and trinary stars, once rough positions of the components are determined from the reconstructed images, these are used as input for a fitting algorithm that computes final position angles, separations and magnitude differences of the components. This is a Fourier-based routine: the power spectrum of the object is formed by Fourier transforming the autocorrelation, and then it is divided by the point source power spectrum. The result for a binary star would be a pure fringe pattern, related to the square of a two-dimensional cosine function. From the separation, orientation, and depth of the fringes, the relative astrometric and photometric parameters for the components can be determined. We use a weighted least-squares fit to the power spectrum in order to arrive at the final parameters for each observation. The methodology of the weighting dates back to Horch et al. (1996), where as described there, we attempt to 

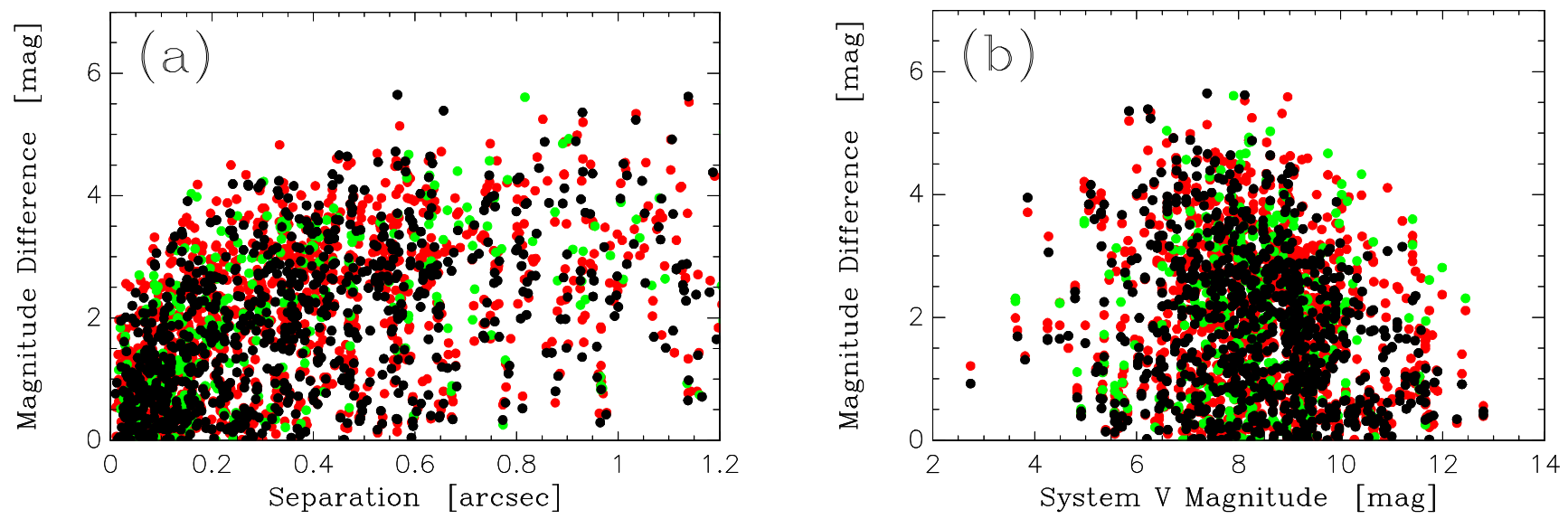

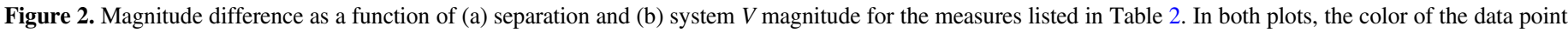

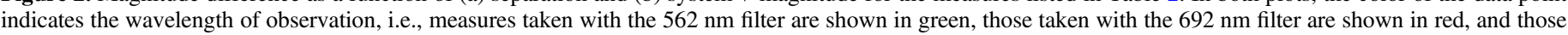
taken in the $880 \mathrm{~nm}$ filter are shown as black.
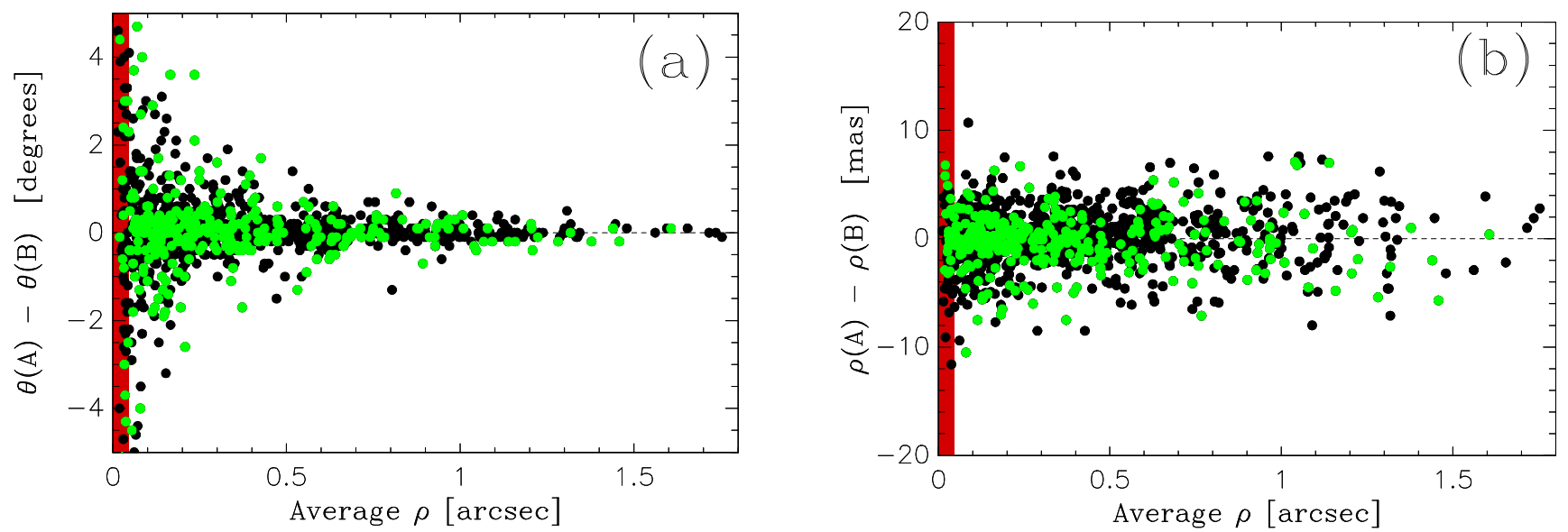

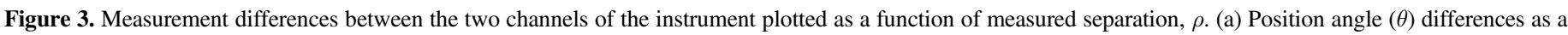

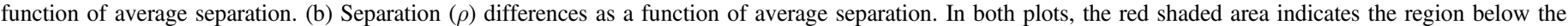

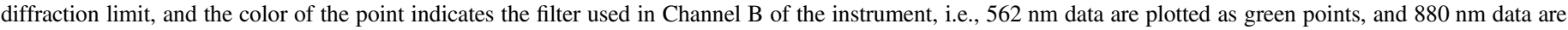
plotted as black points.

approximate a $\chi^{2}$-minimization procedure. In order to do that, one must understand the true noise statistics in the power spectrum, which in turn requires a knowledge of the read noise of the detector and the number of photons per ADU as a function of the electron multiplying gain. We have studied this with our EMCCDs and built this into the current weighting model. More recently, Pluzhnik (2005) has provided a rigorous method for applying similar ideas to speckle data even for non-circular speckle transfer functions; our method is more heuristic in the sense that the variance of points in the power spectrum is estimated from the (properly scaled) data themselves and the final variance needed for calculating $\chi^{2}$ is not an analytical function but instead a smoothed version of the derived variance function in the Fourier plane.

\subsection{Double Star Measures}

Our measures of double stars are found in Table 2. The columns give: (1) the Washington Double Star (WDS) number (Mason et al. 2001), which also gives the R.A. and decl. for the object in 2000.0 coordinates; (2) the Aitken Double Star (ADS) Catalog number, or if none, the Bright Star Catalog (i.e., Harvard
Revised (HR)) number, or if none, the Henry Draper Catalog (HD) number, or if none the Durchmusterung (DM) number of the object; (3) the Discoverer Designation; (4) the Hipparcos Catalog number; (5) the Besselian date of the observation; (6) the position angle $(\theta)$ of the secondary star relative to the primary, with north through east defining the positive sense of $\theta$; (7) the separation of the two stars $(\rho)$, in arcseconds; (8) the magnitude difference $(\Delta m)$ of the pair in the filter used; $(9)$ the center wavelength of the filter; and (10) the full width at half maximum of the filter transmission. The position angle measures have not been precessed from the dates shown. 66 pairs in the table have no previous detection of the companion in the Fourth Catalog of Interferometric Measures of Binary Stars (Hartkopf et al. 2001b); we propose discoverer designations of YSC (YaleSouthern Connecticut) 168-231 here. (Two systems discovered were trinaries.) This continues the collection of YSC discoveries detailed in our earlier papers in this series. In Figure 2, we show two characterizations of the data set as a whole; in panel (a) we plot the magnitude difference obtained as a function of separation, while in panel (b) we plot the same as a function of system apparent $V$ magnitude. 
Table 3

Ephemeris Positions and Residuals Used in the Astrometric Accuracy Study

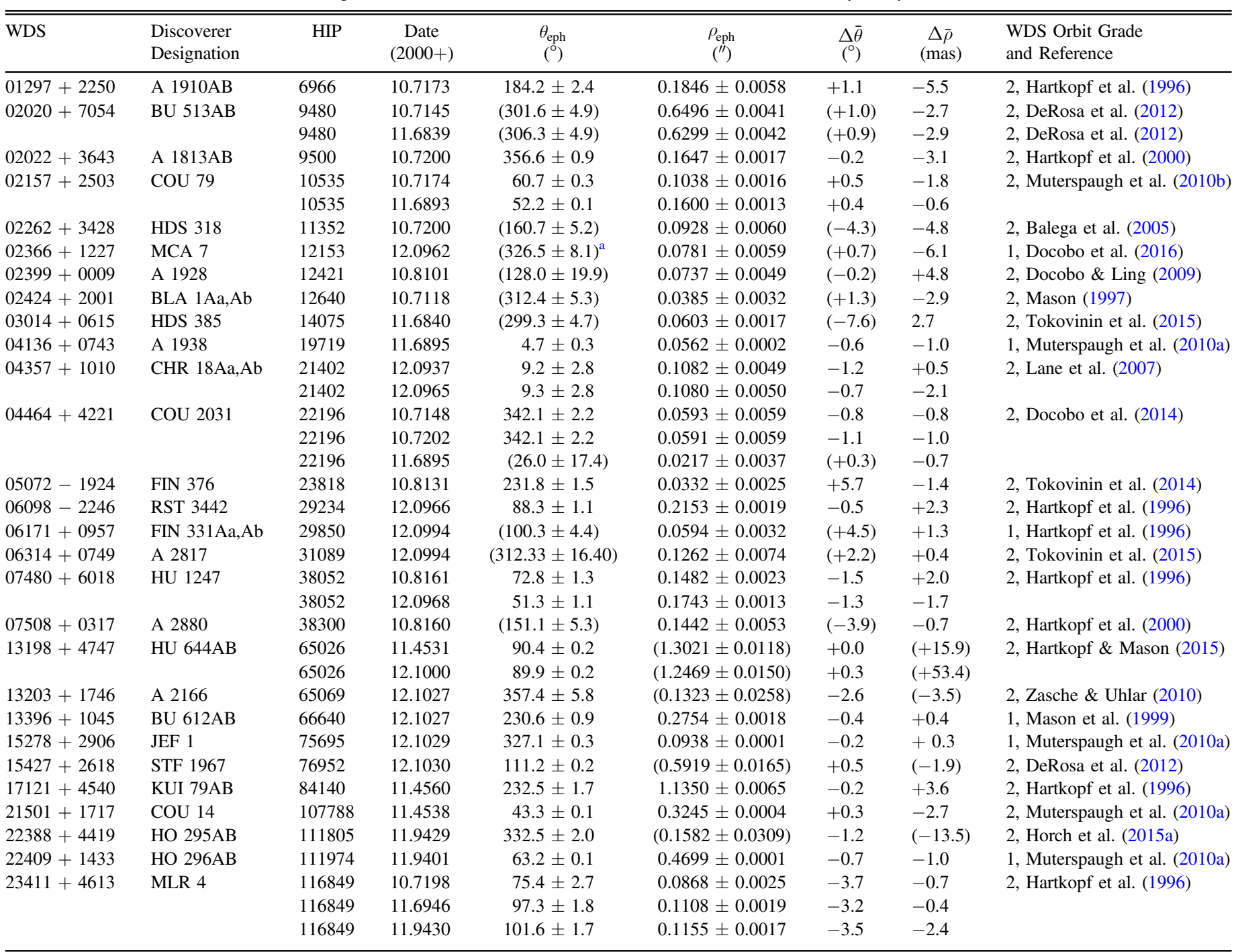

Note.

${ }^{\text {a }}$ Figures in parentheses did not meet the criteria explained in the text for comparison.

\subsubsection{Astrometric Accuracy and Precision}

The astrometric accuracy of the data set is important to establish so that, when used in orbit calculations, a proper weighting can be used. Since the DSSI camera is a two-channel instrument, a fundamental calibration in this regard is a comparison between the results obtained in the channels for each observation. This is shown in Figure 3 for both position angle and separation. All observations in Table 2 are included. The results show that the repeatability between the two channels in position angle is a function of separation, while the repeatability in separation is essentially independent of separation. The average difference between the channels in position angle is $-0.01 \pm 0.04$ degrees, with a standard deviation of $1.51 \pm 0.03$ degrees. In separation, the average difference is $0.06 \pm 0.07$ mas, with a standard deviation of $2.45 \pm 0.05$ mas. As these standard deviation values are the result of the subtraction of two measures that may be assumed to have similar precisions, the precision of individual measures in a single channel can be estimated by dividing these results by a factor of $\sqrt{2}$, resulting in an overall internal precision of $1.07 \pm 0.02$ degrees and $1.73 \pm 0.04$ mas. These numbers set the lower limit of the uncertainty for the data set. If the astrometry of paired measures from the two channels are averaged (which we did not do in Table 2), then there would be a further reduction of a factor of $\sqrt{2}$ in that case, resulting in a separation repeatability of about 1.2 mas, for example, which is comparable to previous data sets we have analyzed from WIYN.

The position angle repeatability is expected to be a function of separation. Position angle measures may be viewed as having a linear measurement uncertainty equivalent to that of the separation measures, but orthogonal in direction (i.e., orthogonal to the vector direction of the separation). In that case, the position angle uncertainty is equal to $\delta \rho / \rho$ in radians, or using the value for $\delta \rho$ obtained here of 1.73 mas and converting to degrees, this is $0.099 / \rho$, where $\rho$ is entered in arcseconds. This is roughly consistent with Figure 3(a). 

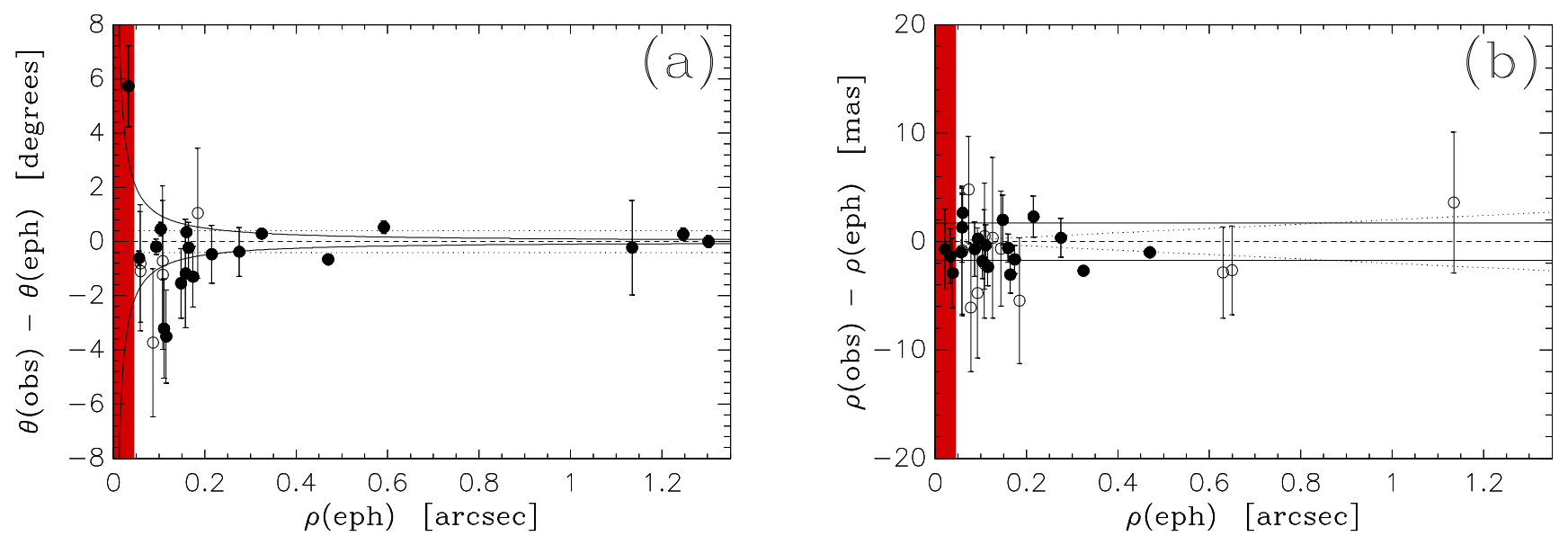

Figure 4. Residual plots for systems that have very well-determined orbits, listed in Table 3. (a) Position angle $(\theta)$ residuals as a function of ephemeris separation for systems with ephemeris uncertainties less than $4^{\circ}$. (b) Separation $(\rho)$ residuals as a function of ephemeris separation for systems with ephemeris uncertainties of less than 8 mas. In both plots, the red shaded area indicates the region below the diffraction limit, the dashed line is the zero line to guide the eye, the solid curves indicate the instrument repeatability as described in the text, and the dotted curves are the uncertainty values from the scale calibration. For position angles, systems with uncertainties in the orbital prediction of less than $2^{\circ}$ are plotted as filled. For separations, systems with ephemeris uncertainties for the epoch of observation of less than 4 mas are shown as filled.

To determine the accuracy of our measures, we examine the results obtained here for stars that already have welldetermined orbits in the literature; specifically, we use systems that have either Grade 1 or Grade 2 orbits in the Sixth Catalog of Visual Binary Star Orbits (Hartkopf et al. 2001a). Only systems that have uncertainty estimates to the orbital elements are considered so that we can propagate errors to estimate the uncertainties in the ephemeris positions, and that the ephemeris uncertainties are less than $4^{\circ}$ and 8 mas respectively. These limits were chosen so that the orbits are of high quality, but also so that the ensemble of systems of sufficient size are statistically meaningful. We then calculate the ephemeris position angle and separation for the system for the epoch of our observations, and use the measures in Table 2 to obtain an observed minus ephemeris residual in both $\rho$ and $\theta$. The systems used for this study appear in Table 3 . The residual plots in position angle and separation are shown in Figure 4. The repeatability uncertainties for single observations derived above, namely $0.099 / \rho$ in position angle and 1.73 mas in separation, are plotted as solid lines. Also plotted as dotted lines are the uncertainties stated in the previous section for the measurement of the pixel scale and orientation. The plots indicate that, for position angle, the internal repeatability dominates the overall uncertainty for separations under 0.3 arcsec, and above that value, the measures are limited by the orientation measures. For the separation measures, uncertainties for separations under $\sim 0.85$ arcsec are dominated by the internal repeatability while larger separations are limited by the the scale measurement. In searching for systematic error by studying average residuals, we find an average offset in position angle in Figure 4(a) of $-0.5 \pm 0.4$ for the entire sample and $-0^{\circ} .3 \pm 0.4$ for the sub-sample of systems with ephemeris uncertainties of less than $2^{\circ}$. For separation, the entire sample has an average residual of $-0.9 \pm 0.4$ mas, with the subset of measures with ephemeris uncertainties of less than 4 mas yielding $-0.6 \pm 0.4$ mas.

Two stars with high-quality orbits in the Sixth Orbit Catalog were not included for the study here. The first is FIN $312=\mathrm{HIP} 12390=\mathrm{HR} 781=\epsilon$ Cet. Although there is a Grade 1 orbit in the literature calculated by Docobo \& Andrade (2013), measures published in 2014 and 2015 have all shown a slightly smaller separation than predicted by about 3 mas, and most since 2005 have the same offset, although the effect appears smaller in the 2005-2013 timeframe. The second is STF $1670=$ HIP $61941=\gamma$ Vir. This well-known visual binary has a Grade 2 orbit calculated by Scardia et al. (2007), but has trended toward smaller separations than predicted since that time; the system has recently passed through periastron and is now rapidly increasing in separation. It is also the case that, at a separation of 1.65 arcsec, the uncertainty in our plate scale determination dominates over the random uncertainties of internal measurement precision. So, our measurements at that separation should be considered less reliable.

Overall, we conclude that there is no evidence of significant systematic offsets in either position angle or separation. At worst, there is a modest offset in separation of a fraction of 1 mas, but this could easily be accounted for in the uncertainty of the orbital elements used and the relatively small number of systems that meet our quality criteria. Therefore, a good measure of the total uncertainty for any measure in Table 2 is obtained by adding the orientation and scale uncertainties in quadrature with the internal random uncertainties obtained from the repeatability study. For example, a system with a separation of $0.1 \operatorname{arcsec}=100$ mas would have a position angle uncertainty of $\sqrt{0.40^{2}+(0.099 / 0.1)^{2}}=1.1$ and a separation uncertainty of $\sqrt{(0.044 / 22) \times 100)^{2}+1.73^{2}}=1.74$ mas, whereas for a system with a separation of $1.0 \operatorname{arcsec}=1000$ mas, the results would be $\sqrt{0.40^{2}+(0.099 / 1.0)^{2}}=0.41$ degrees in position angle and $\sqrt{(0.044 / 22) \times 1000)^{2}+1.73^{2}}=2.64$ mas in separation.

\subsubsection{Photometric Precision}

To judge the photometric precision, we first compute the parameter that in previous papers we have called $q^{\prime}$, which is given by the seeing of the observations multiplied by the separation of the pair. As shown, for example, in Horch et al. (2009), this parameter, in arcseconds squared, should be proportional to the separation divided by the isoplanatic angle, therefore providing a measure of to what degree (in relative terms) the observation is isoplanatic. If an observation lacks 

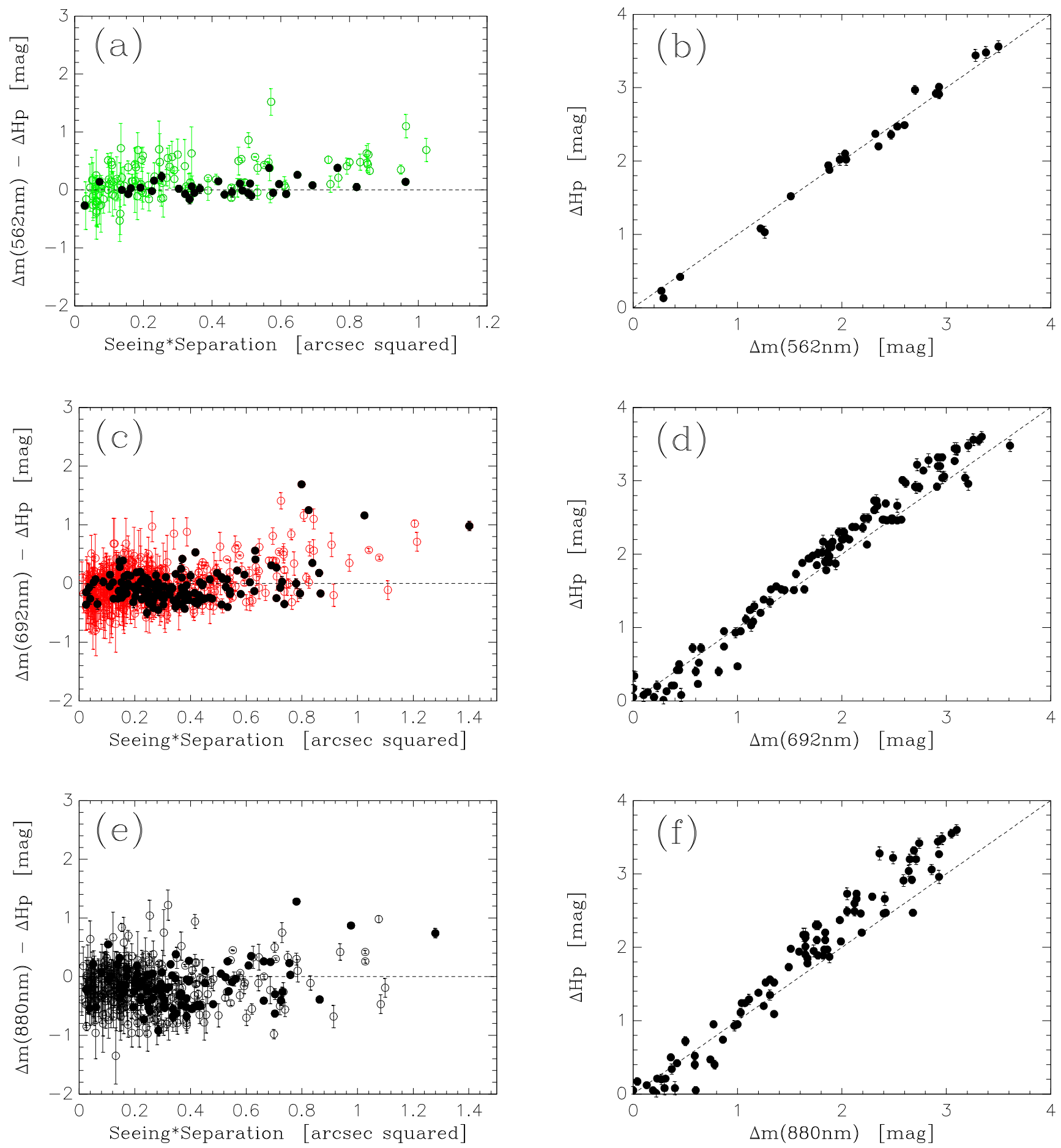

Figure 5. Photometric precision for the data set as a whole. In all plots, Hipparcos measures with uncertainties of less than 0.1 mag are shown as filled black circles, and measures with larger uncertainties are shown as colored open circles, with the color indicating the wavelength used. (a) Differences in $\Delta m$ as a function of seeing time separation for the $562 \mathrm{~nm}$ filter vs. $\Delta H_{p}$. (b) A plot of $\Delta H_{p}$ as a function of $\Delta m$ obtained at $562 \mathrm{~nm}$. (c) The same as panel (a) for the $692 \mathrm{~nm}$ filter. (d) The same as panel (b) for the $692 \mathrm{~nm}$ filter. (e) The same as panel (a) for the $880 \mathrm{~nm}$ filter. (f) The same as panel (b) for the $880 \mathrm{~nm}$ filter.

isoplanicity, one would expect that the magnitude difference obtained from a speckle analysis would be systematically too large.

In Figure 5, we show the differences in $\Delta m$ obtained from our measures in Table 2 versus the Hipparcos measures, $\Delta H_{p}$. Giants, variable stars, and trinary systems are removed from the sample prior to plotting, as are stars with $B-V$ colors greater than 0.6. None of our filters match the Hipparcos $H_{p}$ filter, but it is instructive to see the comparison with each of the filters we used. The closest match is our $562 \mathrm{~nm}$ filter, which has width $\Delta \lambda=40 \mathrm{~nm}$. We see in Figure 5(a) that the average difference $\Delta m-\Delta H_{p}$ is modest, and that the largest differences are found at the highest values of seeing times separation, above approximately $0.55 \operatorname{arcsec}^{2}$. In the case of the redder filters (panels (c) and (e) of the figure), a negative difference is present at low values of $q^{\prime}$, but above $0.55 \operatorname{arcsec}^{2}$, again we 

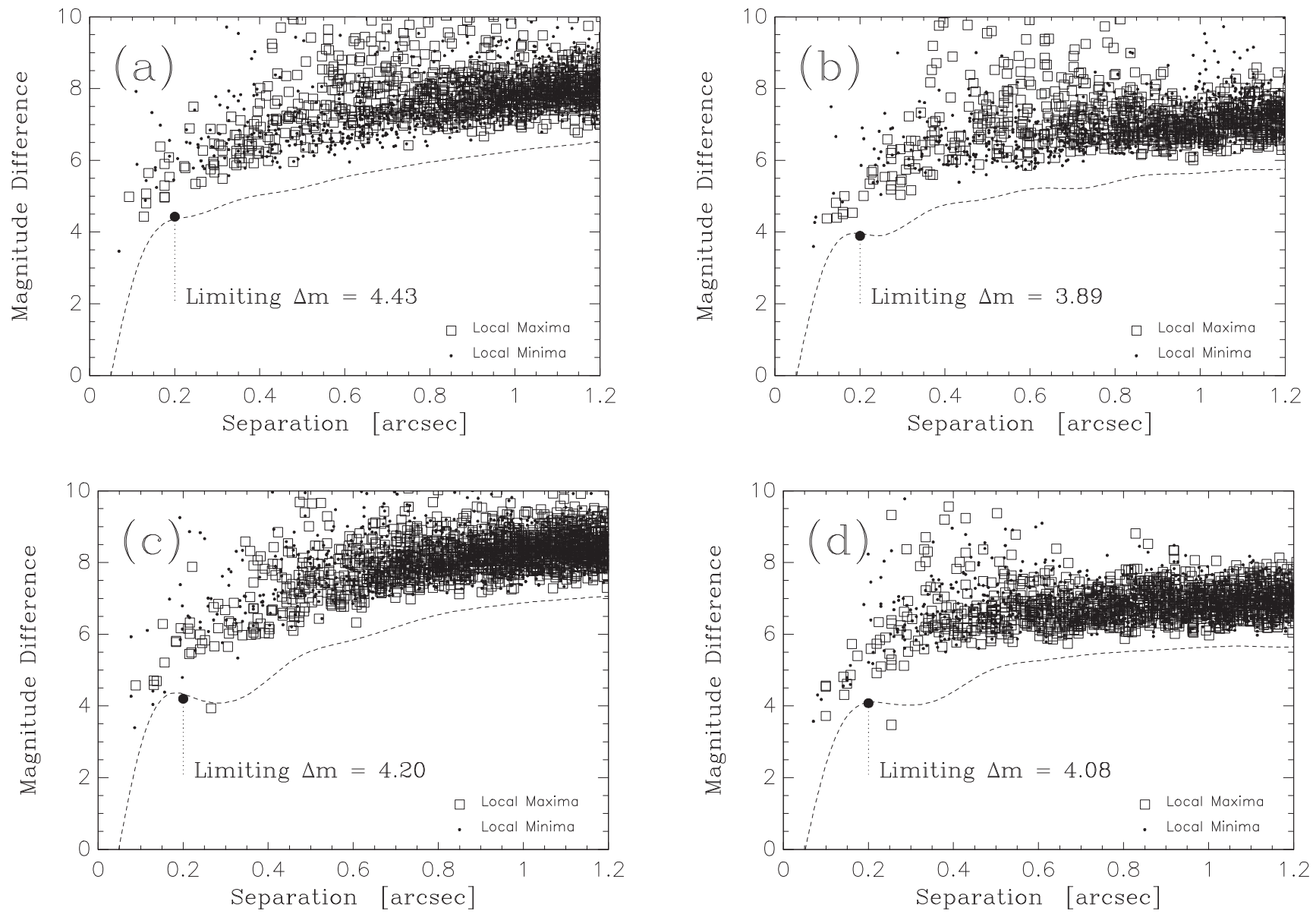

Figure 6. Detection limit plots for HIP 16467, where no companion was found, and HIP 31703, where we report a new component in Table 2. In all cases, squares represent the positions of local maxima in the reconstructed image and dots represent local minima (where the absolute value of the minimum is used). The dashed line represents the 5- $\sigma$ line as a function of separation. (a) HIP 16467 at $692 \mathrm{~nm}$. (b) HIP 16467 at $880 \mathrm{~nm}$. Note that in this case no point lies below the 5- $\sigma$ curve, indicating that no companion was found. (c) YSC $191=$ HIP 31703, at $692 \mathrm{~nm}$. (d) YSC $191=$ HIP 31703, at $880 \mathrm{~nm}$. In this case, a single square lies below the 5- $\sigma$ line in both filters, indicating the detection of a companion at a separation of approximately 0.26 arcsec.

see a rough trend toward large positive offsets. This is expected, since in cases of high isoplanicity (and low $q^{\prime}$ ), redder filters would generate a smaller magnitude difference than the bluer $H_{p}$ filter for main-sequence stars, given the intrinsic color differences of the companions. However, for high values of $q^{\prime}$, the lack of isoplanicity eventually overwhelms that effect and leads to a larger $\Delta m$ regardless of the color difference. In these panels, the data points are divided into two sub-groups depending on the estimated uncertainty of the $\Delta H_{p}$ measure in the Hipparcos Catalog. If less than $0.1 \mathrm{mag}$, then the point is plotted as a filled black circle, and if greater, then as an open circle with the color indicating the filter.

In panels (b), (d), and (f) of Figure 5, we plot the $\Delta H_{p}$ value as a function of our $\Delta m$ for the high-quality subset of measures from panels (a), (c), and (e) respectively. Specifically, these are the measures that have $\delta\left(\Delta H_{p}\right)<0.1 \mathrm{mag}$ and $q^{\prime}<0.55 \mathrm{arcsec}^{2}$. For the $562 \mathrm{~nm}$ filter, which is the closest match to $\Delta H_{p}$, we find an average offset for this subset of $0.01 \pm 0.03 \mathrm{mag}$, with a standard deviation of $0.12 \pm 0.02 \mathrm{mag}$. Some of this uncertainty is due to the Hipparcos measure; the average uncertainty in this sub-sample is $0.052 \mathrm{mag}$. Subtracting this value in quadrature from the standard deviation of the differences, we obtain $0.10 \mathrm{mag}$. Thus, this is the best measure of the uncertainty of our magnitude differences. The other filters show larger deviations from the unitary line and with larger scatter, as expected in the case of a sample of main-sequence stars with a variety of colors. This should not be interpreted as a decrease in photometric precision in these cases, but merely the result of the difference in central wavelength of the filters. We also examined cases in Table 2 where four or more magnitude difference measures appear for a given pair in a single filter, and we computed the standard deviation of each group of observations. The average of these 12 cases, which are mainly observations in the $692 \mathrm{~nm}$ filter, is $\langle\sigma(\Delta m)\rangle=0.09 \pm 0.02 \mathrm{mag}$, in good agreement with the uncertainty derived from the above study. Therefore, we believe the result of uncertainties of typically \pm 0.1 obtained in the $562 \mathrm{~nm}$ filter is probably indicative of the other filters as well.

\subsection{Non-detections}

In a number of cases, we observed stars under good conditions and failed to detect a companion star. The majority of these cases are examples of either Hipparcos suspected double stars or stars found to be double-lined spectroscopic binary stars by the Geneva-Copenhagen spectroscopic survey of G-dwarfs. (For many stars in the latter category, the separation is most likely too small to be measurable from WIYN.) For these cases, we have derived a detection limit estimate as a function of separation using the method described in previous papers. Briefly, we examine annuli in the 
Table 4

5- $\sigma$ Detection Limits for Unresolved Stars

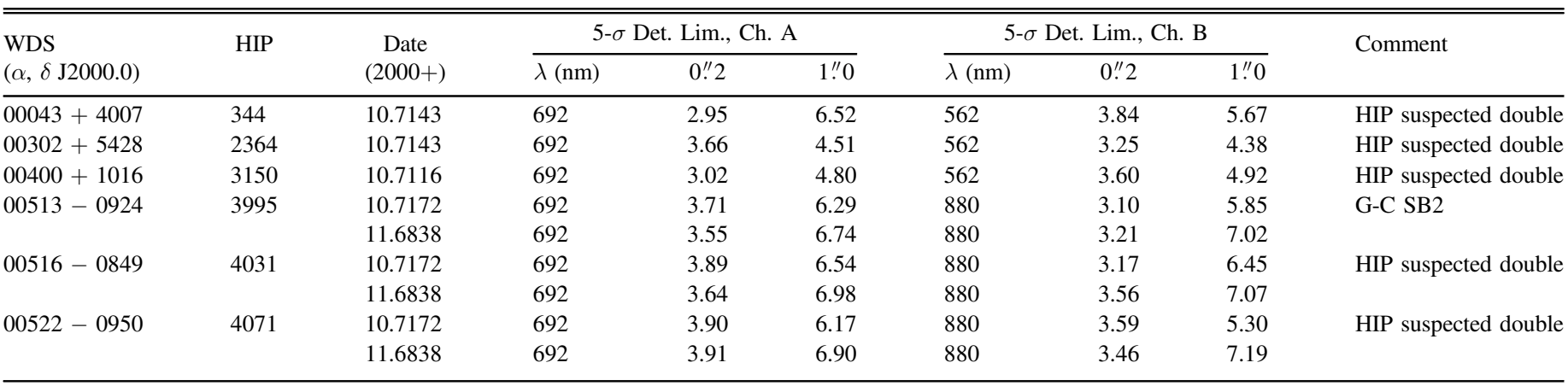

(This table is available in its entirety in machine-readable form.)

Table 5

Preliminary Orbits for Three K-dwarf Systems

\begin{tabular}{llll}
\hline \hline Parameter & HDS 99Aa,Ab & WSI 74Aa,Ab & HDS 2053 \\
\hline HIP & 3493 & 62505 & 71108 \\
Spectrum $^{\mathrm{a}}$ & $\mathrm{K} 7 \mathrm{~V}$ & $\mathrm{~K} 2.5 \mathrm{Vk}$ & $\mathrm{K} 4 \mathrm{~V}$ \\
$\Delta m^{\mathrm{b}}$ & 0.6 & 1.4 & 2.3 \\
$\pi$, mas $^{\mathrm{c}}$ & $31.81 \pm 2.52$ & $41.96 \pm 3.00$ & $24.28 \pm 2.42$ \\
$P$, years & $8.667 \pm 0.065$ & $2.643 \pm 0.012$ & $23.52 \pm 0.36$ \\
$a$, mas & $132.4 \pm 1.2$ & $88 . \pm 10$. & $230.5 \pm 3.4$ \\
$i$, degrees & $171.5 \pm 6.6$ & $59.0 \pm 5.7$ & $103.10 \pm 0.68$ \\
$\Omega$, degrees & $66 . \pm 25$. & $147.7 \pm 2.2$ & $52.15 \pm 0.90$ \\
$T_{0}$, years & $2011.977 \pm 0.023$ & $2016.35 \pm 0.10$ & $2004.49 \pm 0.55$ \\
$e$ & $0.493 \pm 0.010$ & $0.520 \pm 0.047$ & $0.361 \pm 0.025$ \\
$\omega$, degrees & $8 . \pm 26$. & $139 . \pm 10$. & $215 . \pm 11$. \\
$M_{\text {tot }}, M_{\odot}$ & $0.96 \pm 0.23$ & $1.31 \pm 0.53$ & $1.55 \pm 0.48$ \\
\hline
\end{tabular}

Notes.

${ }^{\text {a }}$ As it appears in SIMBAD.

${ }^{\mathrm{b}}$ An average of values appearing in the 4th Interferometric Catalog that have filter center wavelengths between 500 and $600 \mathrm{~nm}$.

${ }^{c}$ From van Leeuwen (2007).

reconstructed images that are centered on the central star and have center radius of a desired value. We determine all local maxima and minima in the annulus, and derive their mean value and standard deviation. We then estimate the detection limit as the mean value of the maxima plus five times the average sigma of the maxima and minima.

The current reduction pipeline produces a curve of this detection limit as a function of separation. Examples are shown in Figure 6. The typical curve rises sharply from the diffraction limit to a "knee" at a separation of $0.15-0.2$ arcsec, then it flattens out somewhat but continues to increase out to the outer limit of the plot, which is 1.2 arcsec. It is interesting to note that this curve is very similar to the envelope of points in Figure 1(a), which is essentially a plot of all detected companions with the same axes. The degree of the flattening above 0.2 arcsec is mainly determined by signal-to-noise ratio and how well the point source chosen for our Fourier deconvolution matches that of the science target, with high signal-to-noise and good matches leading to less flattening, while poor signal-to-noise and/or less perfect matches leading to more flattening. Thus, the curve can be roughly characterized by its value at two points: the knee, and the largest separation point. The curve can then be roughly reconstructed by drawing a line from a magnitude difference of zero at the diffraction limit up to the knee, and a second line from the detection limit at the knee to that at a large separation. In Table 4, we show detection limits determined in this way for 0.2 and 1.0 arcsec for 176 stars for which no companions were found.

\section{Orbits for Three Nearby K-dwarf Binaries}

Much of our binary star speckle work at WIYN to date has been focussed on HDSs within 250 pc of the Solar System, without regard to spectral type. However, there are compelling reasons to refocus attention on a sample of K-dwarfs that are nearby. For G-dwarfs and M-dwarfs, multiplicity studies have either been completed or started (Raghavan et al. 2010; Winters et al. 2015). However, for K-dwarfs, less work has been done and yet for the nearest systems, speckle imaging samples separations that are comparable to the scale of our own Solar System. The main studies enabled by a target list of $\mathrm{K}$-dwarfs are therefore to (a) discover how unusual our Sun is in its stellar solitude, (b) understand how many stars of different types are multiples, (c) provide fundamental statistics that will drive theoretical work in the area of star formation, and (d) provide a list of stars where nearby analogs to the Solar System might be found because they lack stars on planetary formation scales.

Because of these facts, two of us (T.H. and J.W.) identified a sample of K-dwarfs within $50 \mathrm{pc}$ of the solar system using the RECONS ${ }^{14} 25$ pc database and Hipparcos results to $50 \mathrm{pc}$, many of which had not been previously observed with speckle imaging. Although we have begun to systematically observe these stars primarily at the DCT and Gemini, it was also useful to look at which of the stars presented here were serendipitously stars on this list. A total of 48 observations in Table 2 were made of 15 targets on the list of K-dwarfs, including three systems resolved here for the first time: YSC 198, 206, and 208. YSC 198 is actually the inner component of a triple star system, with the tertiary component having separation of $\sim 5$ arcsec. Four stars on the K-dwarf list were unresolved and appear in Table 4. Of all the K-dwarfs observed, three systems had sufficient data in the literature so that, combined with the astrometric data presented here, we were able to attempt first orbit calculations. We discuss these systems below, and orbital parameters are found in Table 5. Interestingly, two of these three systems have a previously known wider component.

\footnotetext{
${ }^{14}$ http://www.recons.org/
} 


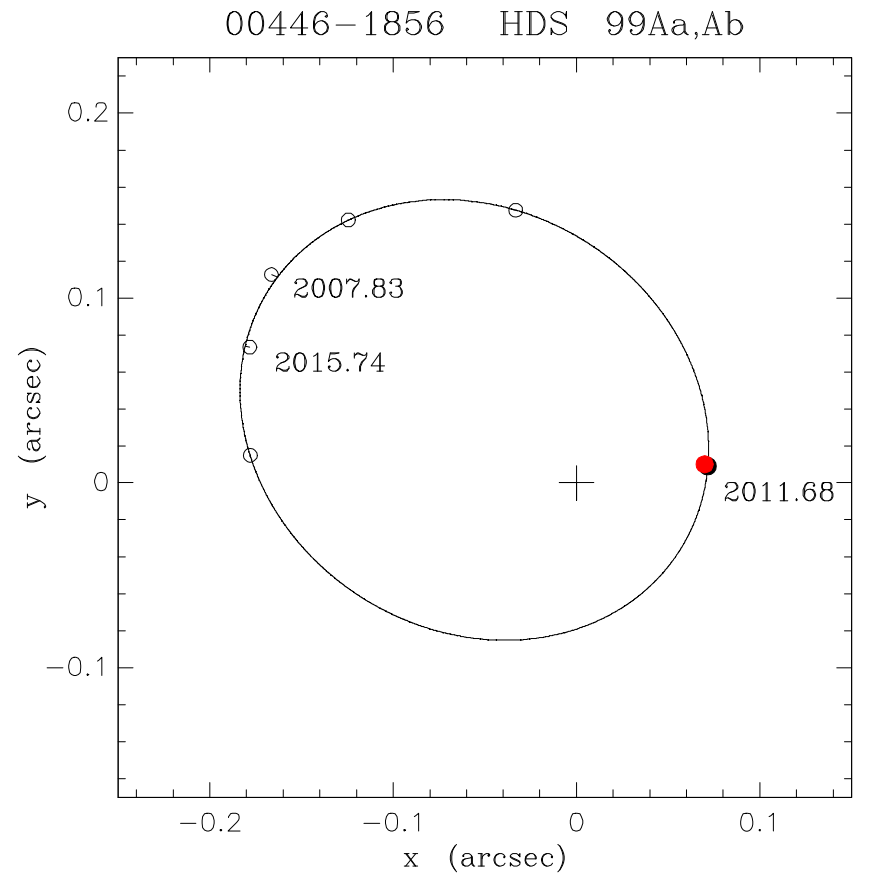

Figure 7. Astrometric data of HDS 99Aa,Ab together with the orbit presented in Table 5. The first and last epoch used in the orbit are labeled, as are the points appearing in Table 2. Points shown as open circles are from data in the 4th Interferometric Catalog; points shown as filled circles indicate data from Table 2, where the color of the point indicates the wavelength of the observation (red being $692 \mathrm{~nm}$ and black being $880 \mathrm{~nm}$ ). In all cases, a line segment is drawn from the ephemeris position to the center of the observational point. North is down and east is to the right.

\section{1. $H D S 99 A a, A b$}

This K7V pair at $31 \mathrm{pc}$ has an orbital period of 8.7 years and semimajor axis of 132.1 mas, which translates to a physical separation of $4.2 \mathrm{au}$. The orbit we calculated did not use the Hipparcos data point due to the relatively short period compared with the length of the Hipparcos mission. The remaining data nonetheless span nearly a full period since 2007. The magnitude difference of the pair is less than 1 , so we may estimate that the pair consists of perhaps a $\mathrm{K} 7$ primary star with a $\mathrm{K} 9$ secondary; this would imply a mass sum in the range of $1.15 M_{\odot}$, using a standard reference such as Schmidt-Kaler (1982). This is consistent with the mass sum calculated from the orbital parameters shown in Table 5, which already has a relatively small uncertainty. This system forms the inner pair of the common proper motion double LDS 3195AB, which has separation of $141 \mathrm{arcsec}$ (or $4450 \mathrm{au}$ ). Our orbit is shown in Figure 7.

\subsection{WSI $74 A a, A b$}

The SIMBAD ${ }^{15}$ database shows the spectral type of this pair to be $\mathrm{K} 2.5 \mathrm{~V}$, which forms the inner system of HDS $1795 \mathrm{AB}$. Given the magnitude difference of approximately 1.4, we estimate that this is a $\mathrm{K} 2 \mathrm{~V}+\mathrm{K} 6 \mathrm{~V}$ system, implying a mass sum of $1.38 M_{\odot}$, based again on data in Schmidt-Kaler (1982). While the orbital elements do not permit anything more than a very rough mass sum to be inferred at this point, it is nonetheless consistent with this value. We find a period of 2.6

\footnotetext{
15 http://simbad.u-strasbg.fr/simbad
}

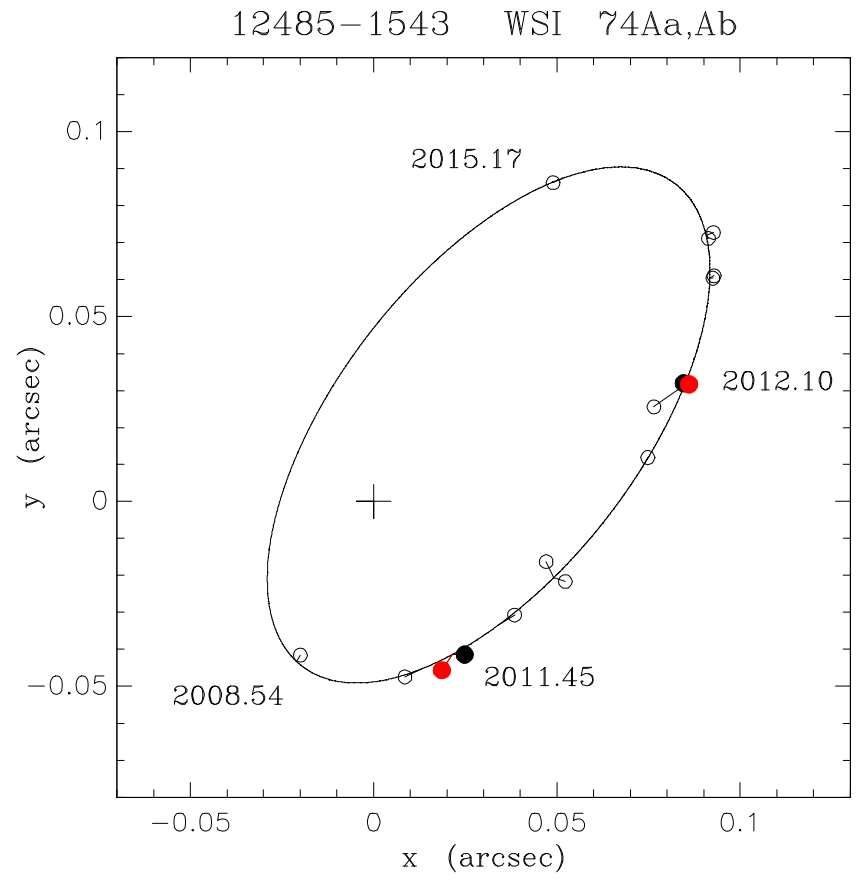

Figure 8. Astrometric data of WSI 74Aa, $\mathrm{Ab}$ together with the orbit presented in Table 5. The first and last epoch used in the orbit are labeled, as are the points appearing in Table 2. Points shown as open circles are from data in the 4th Interferometric Catalog; points shown as filled circles indicate data from Table 2, where the color of the point indicates the wavelength of the observation (red being $692 \mathrm{~nm}$ and black being $880 \mathrm{~nm}$ ). In all cases, a line segment is drawn from the ephemeris position to the center of the observational point. The quadrant of the 2011 observations shown in Table 2 is inconsistent with other existing measures; we have reversed that here and in the orbit calculation. North is down and east is to the right.

years and a semimajor axis of 88 mas (2.1 au). The wider component, which was out of our small field of view, is at a projected separation of $2.7 \operatorname{arcsec}(64 \mathrm{au})$. The 4th Interferometric Catalog contains several non-detections by Tokovinin, but these are at or below the diffraction limit during the epochs in question for the orbit presented here. The plot of the orbit is shown in Figure 8.

\section{3. $\operatorname{HDS} 2053$}

This system has the largest magnitude difference of the three systems under consideration here at approximately $2.3 \mathrm{mag}$, so despite relatively few data points to work with for the orbit calculation, the determination of the quadrant for each observation should not be in question. We calculated this orbit with and without the Hipparcos point, given the orbital period. We present elements without the Hipparcos point in Table 5; when the point is included, the period is shorter by approximately 4 years, but the semimajor axis is similar. However, looking at the position of the Hipparcos point on the orbit, it is clear that there is the potential to derive a systematically low separation if averaging the position of the secondary over the lifetime of the Hipparcos mission. The resulting total mass estimate is $1.5 \pm 0.5 M_{\odot}$. We estimate that this system consists of a K4V primary star with a M1V secondary, so that the expected total mass would be $\sim 1.2 M_{\odot}$. The semimajor axis that we derive using the revised Hipparcos parallax is $9.5 \mathrm{au}$. We show our orbit in Figure 9. 


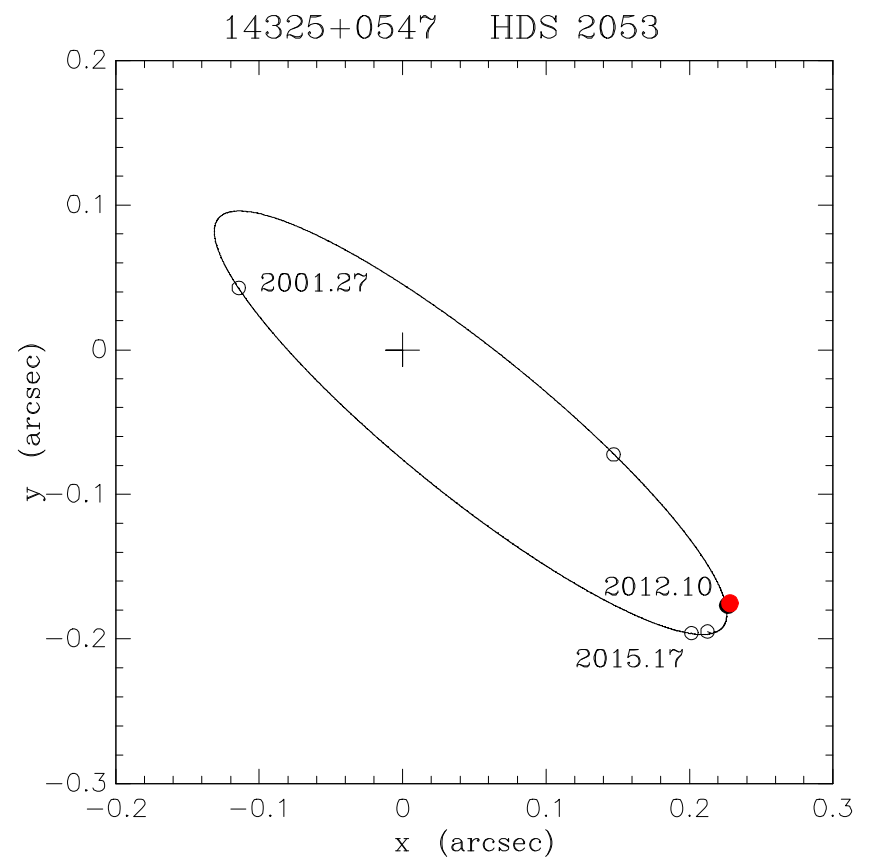

Figure 9. Astrometric data of HDS 2053 together with the orbit presented in Table 5. The first and last epoch used in the orbit are labeled, as are the points appearing in Table 2. Points shown as open circles are from data in the 4th Interferometric Catalog; points shown as filled circles indicate data from Table 2, where the color of the point indicates the wavelength of the observation (red being $692 \mathrm{~nm}$ and black being $880 \mathrm{~nm}$ ). In all cases, a line segment is drawn from the ephemeris position to the center of the observational point. North is down and east is to the right.

\section{Summary}

We have presented the results of over 2800 observations of double stars and suspected double stars using the dual-channel speckle imaging camera, DSSI, when it was resident at the WIYN Observatory at Kitt Peak during 2010-2012. The astrometric precision appears to be in line with previous papers in this series, generally $\sim 2$ mas in separation and $\sim(0.1 / \rho)^{\circ}$ in position angle, where $\rho$ is entered in arcseconds. The photometric precision is generally about 0.1 mag per observation. In cases where there is evidence for a lack of isoplanicity or if the secondary falls near the edge of the frame, we have not reported magnitude differences. 176 additional objects showed no evidence of a companion and in these cases we have derived 5- $\sigma$ detection limits at separations of 0.2 and 1.0 arcsec.

The data presented here, along with existing relative astrometry already in the literature, permitted the calculation of preliminary orbits for three K-dwarf binaries at distances of $24-42 \mathrm{pc}$. The periods ranged from 2.6 to 23.5 years, and initial mass estimates from the orbital elements are consistent with the expected theoretical values for stars of that spectral type. These serve as examples for the potential of sustained, well-calibrated astrometry efforts on such systems.

The authors would like to thank all of the excellent staff at the WIYN telescope for their help during our observing runs. We were privileged to work with professionals of such dedication and skill. We used the SIMBAD database, the Washington Double Star Catalog, the Fourth Catalog of Interferometric Measures of Binary Stars, and the Sixth Orbit
Catalog in the preparation of this paper. We gratefully acknowledge the role of the Kepler Science Office in upgrading DSSI to the two-EMCCD mode used here, support from National Science Foundation grant AST-0908125, which funded the observations discussed here, and grant AST1517824, which funded the completion of the analysis and publication of this work.

\section{References}

Appourchaux, T., Antia, H. M., Ball, W., et al. 2015, A\&A, 582, A25 Balega, I. I., Balega, Y. Y., Gasanova, L. T., et al. 2013, AstBu, 68, 53 Balega, I. I., Balega, Y. Y., Hofmann, K.-H., et al. 2005, A\&A, 433, 591 Ciardi, D. R., Beichman, C. A., Horch, E. P., \& Howell, S. B. 2015, ApJ, 805,16

Crossfield, I. J., Ciardi, D. R., Petigura, E. A., et al. 2016, ApJS, 226, 7 DeRosa, R. J., Patience, J., Vigan, A., et al. 2012, MNRAS, 422, 2765

Docobo, J. A., \& Andrade, M. 2013, MNRAS, 428, 321

Docobo, J. A., Griffin, R. F., Tamazian, V. S., et al. 2014, MNRAS, 444, 3641 Docobo, J. A., \& Ling, J. F. 2009, AJ, 138, 1159

Docobo, J. A., Tamazian, V. S., Balega, Y. Y., \& Melikian, N. D. 2010, AJ, 140,1078

Docobo, J. A., Tamazian, V. S., Malkov, O. Y., et al. 2016, MNRAS, 459, 1580

ESA 1997, The Hipparcos and Tycho Catalogues (ESA: Noordwijk) ESA SP 1200

Everett, M. E., Barclay, T., Ciardi, D. R., et al. 2015, AJ, 149, 55

Furlan, E., Ciardi, D. R., Everett, M. E., et al. 2017, AJ, 153, 71

Hartkopf, W. I., \& Mason, B. D. 2015, AJ, 150, 136

Hartkopf, W. I., Mason, B. D., \& McAlister, H. A. 1996, AJ, 111, 370

Hartkopf, W. I., Mason, B. D., McAlister, H. A., et al. 2000, AJ, 119, 3084

Hartkopf, W. I., Mason, B. D., \& Worley, C. E. 2001a, AJ, 122, 3472

Hartkopf, W. I., McAlister, H. A., \& Mason, B. D. 2001b, AJ, 122, 3480

Hirsch, L. A., Ciardi, D. R., Howard, A. W., et al. 2017, AJ, 153, 117

Hoffleit, E. D., \& Jaschek, C. 1982, The Bright Star Catalogue (New Haven, CT: Yale Univ. Observatory)

Horch, E. P., Bahi, L. A. P., Gaulin, J. R., et al. 2012, AJ, 143, 10

Horch, E. P., Dinescu, D. I., Girard, T. M., et al. 1996, AJ, 111, 1681

Horch, E. P., Gomez, S. C., Sherry, W. H., et al. 2011a, AJ, 141, 45

Horch, E. P., Howell, S. B., Everett, M. E., \& Ciardi, D. R. 2014, ApJ, 795, 60

Horch, E. P., Ninkov, Z., van Altena, W. F., et al. 1999, AJ, 117, 548

Horch, E. P., van Altena, W. F., Demarque, P., et al. 2015a, AJ, 149, 151

Horch, E. P., van Altena, W. F., Howell, S. B., et al. 2011b, AJ, 141, 180

Horch, E. P., van Belle, G. T., Davidson, J. W., Jr., et al. 2015b, AJ, 150, 151

Horch, E. P., Veillette, D. R., Baena Gallé, R., et al. 2009, AJ, 137, 5057

Howell, S. B., Everett, M. E., Horch, E. P., et al. 2016, ApJ, 829, 2

Howell, S. B., Everett, M. E., Sherry, W. H., et al. 2011, AJ, 142, 19

Kane, S. R., Howell, S. B., Horch, E. P., et al. 2014, ApJ, 785, 93

Lane, B. F., Muterspaugh, M. W., Fekel, F. C., et al. 2007, ApJ, 669, 1209

Lohmann, A. W., Weigelt, G., \& Wirnitzer, B. 1983, ApOpt, 22, 4028

Mason, B. D. 1997, AJ, 114, 808

Mason, B. D., Douglass, G. G., \& Hartkopf, W. I. 1999, AJ, 117, 1023

Mason, B. D., Wycoff, G. L., Hartkopf, W. I., Douglass, G. G., \& Worley, C. E. 2001, AJ, 122, 3466

Meng, J., Aitken, G. J. M., Hege, K., \& Morgan, J. S. 1990, JOSAA, 7, 1243 Muterspaugh, M. W., Hartkopf, W. I., Lane, B. F., et al. 2010a, AJ, 140, 1623

Muterspaugh, M. W., Lane, B. F., Kulkarni, S. R., et al. 2010b, AJ, 140, 1657

Nordström, B., Mayor, M., Andersen, J., et al. 2004, A\&A, 419, 989

Pickles, A. J. 1998, PASP, 110, 863

Pluzhnik, E. A. 2005, A\&A, 431, 587

Raghavan, D., McAlister, H. A., Henry, T. J., et al. 2010, ApJS, 190, 1

Scardia, M., Argyle, R. W., Prieur, J.-L., et al. 2007, AN, 328, 146

Schmidt-Kaler, T. 1982, in Stars and Star Clusters, Group 6, Vol. 2b, ed.

K. Schaefers \& H.-H. Voigt (Berlin: Springer), 1

Teske, J. K., Everett, M. E., Hirsch, L., et al. 2015, AJ, 150, 144

Tokovinin, A., \& Cantarutti, R. 2008, PASP, 120, 170

Tokovinin, A., Mason, B. D., \& Hartkopf, W. I. 2014, AJ, 147, 123

Tokovinin, A., Mason, B. D., Hartkopf, W. I., et al. 2015, AJ, 150, 50

van Leeuwen, F. 2007, A\&A, 474, 653

Winters, J. G., Henry, T. J., Lurie, J. C., et al. 2015, AJ, 149, 5

Zasche, P., \& Uhlar, R. 2010, A\&A, 519, A78 